\title{
Enfacing a female reduces the gender-science stereotype in males
}

\author{
Xingyu Zhang ${ }^{1} \cdot$ Bernhard Hommel $^{2,3} \cdot \mathrm{Ke} \mathrm{Ma}^{1}$ (1) \\ Accepted: 2 January 2021 / Published online: 21 January 2021 \\ (C) The Psychonomic Society, Inc. 2021
}

\begin{abstract}
The enfacement illusion refers to the illusory perception that features of another face that moves in synchrony with one's own facial movements become part of one's own body. Here, we tested whether males whose facial movements are synchronized with a virtual female face exhibit a less pronounced implicit gender-science stereotype than males whose movements are not synchronized. Results show that illusory ownership and agency of the face with opposite gender was successfully induced, and that synchrony significantly reduced the implicit gender-science stereotype as compared with nonsynchrony. Our findings are in line with previous demonstrations about being synchronized with other individuals facilitates feature migration - that is, the integration of their features into one's self-representation.
\end{abstract}

Keywords Virtual enfacement illusion $\cdot$ Gender-science stereotype $\cdot$ Implicit attitude $\cdot$ Feature migration

\section{Introduction}

Body ownership refers to the experience that oneself is the owner of one's body (Jeannerod, 2003). While this experience seems to be self-evident, the experience of body ownership was shown to be quite malleable and much more flexible than people would have imagined in a number of studies (Ramachandran, 1998). For example, in the rubber-hand illusion (RHI), participants are asked to watch a rubber hand in front of them while their corresponding real hand is hidden from their view. If, then, both real hand and rubber hand are brushed simultaneously, participants felt to gain ownership over the rubber hand (Botvinick \& Cohen, 1998). By replacing the rubber hand with a virtual hand that can be voluntarily operated by participants, a very similar illusion emerges: the virtual hand illusion (VHI), also consisting of the feeling to gain ownership over the virtual hand (e.g., Ma \& Hommel, 2013). Research on the flexibility of body representation has

Bernhard Hommel

bh@bhommel.onmicrosoft.com

Ke Ma

psyke1@swu.edu.cn

1 Key Laboratory of Personality and cognition, Faculty of Psychology, Southwest University, Beibei, Chongqing, China

2 Institute for Psychological Research \& Leiden Institute for Brain and Cognition, Leiden University, Leiden, the Netherlands

3 Department of Psychology, Shandong Normal University, Jinan, China also extended to ownership illusions involving other parts of the body, such as the face (Tsakiris, 2008). Tsakiris had participants watch a video in which another face was shown in a situation similar to watching one's own reflection in a mirror. Then, the cheeks of the participant and the viewed face were brushed synchronously or asynchronously, with the result that synchronously stimulated participants identified more facial features of the viewed face as their own-the self-other boundary was thus blurred.

Observations of this degree of flexibility regarding the way people represent themselves do not fit with the traditional assumption of a rather permanent body image, but support the assumption that bottom-up cues play an important role in self-representation (Botvinick \& Cohen, 1998). As Hommel and colleagues (Hommel, 2013, 2018; Ma, Sellaro, Lippelt, \& Hommel, 2016) have suggested, people may represent themselves and others by means of event files - that is, bindings of features perceived to belong to (i.e., to covary with other features of) oneself or others. Just like in the case of Gestalt perception, the degree to which features are integrated into the same event file or segregated into different ones may thus depend on the covariation of features across time and space and on the present processing mode, which may favor integration or segregation (Hommel \& Colzato, 2017). This approach suggests that if covariation is consistent, as in synchrony conditions, and/or the processing mode favors integration, features of nominally different events may be confused and integrated into the same event file-similarly to the demonstrations of "feature migration" in visual identification tasks (Treisman \& Gelade, 1980). Social feature migration using the 
enfacement illusion has indeed been observed. Ma et al. (2016) presented participants with virtual faces that at some point started to smile or continue to show a neutral expression, after the head movements of the participants were or were not synchronized with the head movements of the virtual face. Participants showed explicit and implicit signs of improved mood after being exposed to a smiling virtual face, but only if they have previously been synced with it. This shows that the feature "smile" migrated from the virtual agent to the participant, if the virtual and the real face showed sufficient covariation. Along the same lines, Ma, Sellaro, and Hommel (2019) showed that humans perform more poorly in an intelligence test after having watched the human face that they were synced with morphing into the face of an ape. At the same time, however, this experience made participants to believe more strongly that animals have emotions, suggesting that synchrony facilitated "feature migration" in terms of intelligence and emotional attribution from the representation of the ape to the representation of the participant himself or herself.

The observation of social feature migration suggests that synchronizing with other individuals might have interesting effects on social cognition, and there is increasing evidence supporting this expectation (Maister, Slater, Sanchez-Vives, \& Tsakiris, 2015). For example, the enfacement illusion has been shown to affect perceived interpersonal similarity and social attitudes, including attraction toward the other, conformity behavior, social inference about the personality, and selfother integration (Mazzurega, Pavani, Paladino, \& Schubert, 2011; Paladino, Mazzurega, Pavani, \& Schubert, 2010), and the illusion has been shown to be positively correlated with participants' empathic traits (Sforza, Bufalari, Haggard, \& Aglioti, 2010). Of particular importance for our present study, researchers found that implicit attitudes can be affected by inducing the RHI (Maister, Sebanz, Knoblich, \& Tsakiris, 2013), the enfacement illusion (Jeong, Chang, \& Kim, 2019), or the virtual full body illusion (Peck, Seinfeld, Aglioti, \& Slater, 2013). For example, Maister et al. (2013) induced the RHI of Caucasian participants by means of a black rubber hand, and measured the participants' implicit racial attitudes towards African American people by means of an implicit association test (IAT; Greenwald, Nosek, \& Banaji, 2003). The RHIrelated body-ownership score significantly predicted the changes of implicit racial attitudes, showing that stronger ownership illusions led to a more pronounced reduction of the negative attitude towards African Americans. Similarly, Peck et al. (2013) found that synchrony with an African American virtual body reduced the implicit racial bias. These observations suggest that synchronizing individuals with targets of negative stereotypes might help to reduce these stereotypes.

In the present study, we extended this logic to the common gender-science stereotype. This stereotype basically considers the study of science-related disciplines (STEM:
Science, Technology, Engineering, and Math; see Smyth \& Nosek, 2015) as less fitting or appropriate for females, who in turn are perceived to fit better with disciplines related to social sciences or the humanities. The gender-science stereotype is rather consistent across age and culture (Cvencek, Meltzoff, \& Greenwald, 2011; Nosek et al., 2009; Nosek, Greenwald, \& Banaji, 2005), especially if being measured implicitly (rather than explicitly) with the IAT (see below for procedural details). The stereotype is also very widespread among Chinese students (Dong, 2019; Wang, 2010; Xu, 2012). Whereas the gender-science stereotype might not be as threatening for the targeted groups as racial biases, it is considered to undermine the motivational state of female students and to affect the study performance of male and female students (Vick, Seery, Blascovich, \& Weisbuch, 2008). The stereotype also influences the choice and development of male and female majors in the future (Cheryan, Master, \& Meltzoff, 2015), with obviously unwanted effects on personal fulfillment and societal productivity.

In the present study, we tested whether the gender-science stereotype can be reduced by synchronizing individuals with a virtual member of the group targeted by this stereotype. To do so, we presented male students with the virtual face of a female, and had the head of the virtual female move synchronously or asynchronously with the head of the human participant. The idea was that synchronizing movements would increase the perceived similarity between male and female, and thus reduce the stereotype based on this discrepancy. The expectation that inducing synchrony would reduce the perceived gender discrepancy was fueled by a recent study of Tacikowski, Fust, and Ehrsson (2020). These authors did not only show that participants perceive ownership of the synchronized body of a virtual agent of the opposite gender, but also found that this condition induced a more balanced perception of both genders and less gender-stereotypical beliefs.

\section{Experiment}

We adopted the basic experimental setup and design used by Ma et al. (2016). Participants were randomly assigned to a synchrony or asynchrony group. In the synchrony group, participants were presented with a virtual face that moved in synchrony with their own facial movements, whereas the movements of the virtual face were delayed by 3 seconds in the asynchrony group. We used Likert scales to assess perceived ownership and agency regarding the virtual face and an IAT paradigm to assess the implicit gender-science stereotype, following Maister et al. (2013); we did not assess participants' explicit attitude regarding gender and science to avoid possible effects of social desirability and demand characteristics. 


\section{Method}

\section{Participants}

All participants enrolled in this study were male. The reason was that though both males and females showed genderscience stereotypes in previous studies, this effect is usually stronger in males than in females (Sanchis-Segura, Aguirre, Cruz-Gómez, Solozano, \& Forn, 2018; Smyth \& Nosek, 2015; Steegh, Höffler, Höft, \& Parchmann, 2020). Especially important for our study, this gender difference is particularly strong in the population we studied - Chinese students - in which stronger gender-science stereotypes in males than in females were demonstrated for both explicit (Dong, 2019) and implicit measures (Wang, 2010).

Ninety-seven college students participated - 49 (mean age $=21.49$ years, $S D=1.31$, range: $19-25$ years) in the synchrony group and 48 (mean age $=21.67$ years, $S D=1.43$, range: 19-24 years) in the asynchrony group. Sample size was determinate a priori by conducting a power analysis using G*Power (Faul, Erdfelder, Lang, \& Buchner, 2007), specifying a medium effect size $(f=0.25)$, and the power $(1-\beta)$ was approximately 0.80 . All the participants had normal vision or corrected vision and did not feel uncomfortable or dizzy in the virtual reality environment. All participants were righthanded.

\section{Setup}

The experimental setup was as in Ma et al. (2016), with the following modifications. We used the software Facegen to generate a virtual 3D model with an average Asian female face. We then imported this virtual face into the virtual reality (VR) environment, which was an empty virtual room designed with the software Vizard. By wearing HTC head mounted displays (HMDs), participants were immersed into this virtual room, as Fig 1 shows. Participants' real head position (which is the same as the HMD's position) coincided with the position of virtual face. In front of the virtual face, we also imported a virtual mirror in this virtual room; thus, participants could see the reflection of the virtual face through the virtual mirror.

\section{Materials}

Ownership and agency questionnaire This questionnaire was adopted from the one used in Ma et al. (2016). It consisted of five items, with three items assessing ownership (the subjective experience that the virtual face reflected in the mirror is integrated into one's own body representation), and two items assessing agency (one's experience of control over the virtual face). Participants were asked to rate each statement on a 7point Likert scale, in which 1 indicated strong disagreement, 4 uncertainty, and 7 strong agreement with the statement. We averaged across the first three items to create the "ownership score" and across the last two items to obtain the "agency score." The specific items were as follows:

Q1. I think the face in front of me is my face.

Q2. I feel like I am looking at a mirror, the face in front of me is the reflection of the mirror on my face.

Q3. I feel the face in front of me is more and more like my own face.

Q4. I think the movement of the face in front of me is caused by me.

Q5. I feel that I can control the movement of the face in front of me, as if it was obeying my will.

Implicit association test (IAT) The task was designed according to the classic IAT paradigm of Greenwald, McGhee, and Schwartz (1998). The set of stimuli consisted of 12 attribute words and 12 concept words. The names and discipline nouns were all adopted from the materials used in $\mathrm{Xu}$ (2012) and Smyth and Nosek (2015). Names were typical Chinese female (Zhang Qianqian, Zhou Huijuan, Ma Yuling, Zhou Na, Wang Fang, and Zhao Li) and male (Wang Kai, Zhang Liang, Chen Jun, He Xiaobo, Zhao Zhiyong, and Li Jiangang) names. Discipline words were the labels of humanities/science majors (non-STEM majors were humanities, philosophy, linguistics,
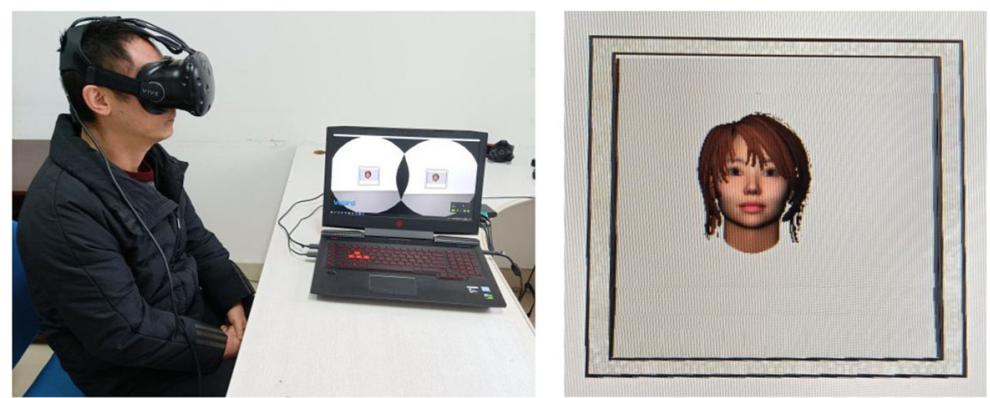

Fig. 1 Left panel: The experiment setup, participants wear the HMD, immerse in a virtual room, viewed the virtual mirror and the virtual face as a reflection of their own face through the mirror. Right panel: The viewed virtual female face by participants 
arts, history, and law; STEM majors were mathematics, physics, chemistry, biology, engineering, and computer science). The task consisted of five blocks: the first, second, and fourth blocks were training blocks of 24 trials each; and the third and fifth blocks were test blocks of 84 trials each.

In the first training block, participants were asked to categorize male and female names by pressing a left and right button (the " $\mathrm{f}$ " and " $\mathrm{j}$ " buttons, respectively, on the computer keyboard). Half of the participants pressed the left button in response to male names and the right button in response to female names, whereas the remaining participants received the opposite mapping. In (training) Block 2, participants used the same response buttons to categorize major words as either science or liberal arts. In (training) Block 4, participants were to discriminate between male and female names, as they did in Block 1, but the response buttons assigned to the target concepts were switched. In Block 3, Block 5, the gender names discrimination and major attributes discrimination tasks were combined. In one of them, male names shared the same response button as science, and female names the same response button as liberal arts (i.e., congruent block). In the other block, male names were associated with liberal arts and female names with science (i.e., incongruent block). The order of the congruent and incongruent blocks, and the pressed button, were all counterbalanced across participants. In each trial, the stimulus was presented until the participant responded or until 4,000 $\mathrm{ms}$ had passed, and trials were separated by a blank of $750 \mathrm{~ms}$. Participants were instructed to respond as quickly and accurately as possible.

\section{Procedure}

First, participants completed the preexperimental IAT (preIAT) when they arrived in the lab. Second, they took on the HMD and experienced a 2-minute inducement phase, during which they were asked to keep their heads rotating or moving and pay attention to the (virtual) facial movement in the (virtual) mirror. Participants in the synchronization group would see the virtual facial movements in the mirror moving synchronously with their real head movement, and participants in the asynchrony group would see the virtual facial movements moving with a 3-s delay relative to their real head movement. To prevent participants from passively following the virtual facial movement in the mirror, we reminded participants to keep moving their head freely and voluntarily. After 1 minute of free movement, participants would be asked general questions, such as giving a simple description of the faces in the mirror (which could be facial features or hair or any other thing) or briefly describing how they feel when moving their heads (especially whether they felt dizzy in the VR environment). Importantly, whenever participants uttered something in the synchrony condition, the VR script made the mouth of the virtual face open and close according to the recorded sound volume - which was thought to further increase the perceived synchrony between the participant and virtual face. The mouth of the virtual face did not open or close in the asynchrony condition. This period took another minute, during which participants could still freely move their head.

After the VR exposure phase and the general questions, the ownership and agency questionnaire was presented in the virtual environment with HMD on. Participants read the questionnaire and orally reported the chosen ratings to each question to the experimenter. Finally, participants took off the HMD and completed the post-experimental IAT ( ${ }_{\text {post }} \mathrm{IAT}$ ).

\section{Results}

We ran both two-tailed independent $t$ tests and nonparametric Wilcoxon tests on the questionnaire data, with synchrony as a within-participants factor. Given that results were the same, we only report the $t$-test results for all measurements.

\section{Ownership-agency questionnaire}

Independent-sample $t$ tests compared the ownership and agency scores of the synchrony and the asynchrony group. Results showed that both the ownership and the agency score was significantly higher in the synchrony than in the asynchrony group, $t(95)=5.05, p<.001, d=1.03 ; t(95)=15.07, p<.001$, $d=3.05$, respectively (see Table 1 ). Hence, the enfacement illusion was successfully induced. Ownership and agency scores correlated significantly and positively, $r=.54, p$ $<.001, N=97$, in one-tailed Spearman tests.

\section{Gender-science IAT}

Following previous studies (Greenwald et al., 2003; Sellaro et al., 2015), IAT $d$ scores were calculate according to the following method: (1) reaction times (RTs) in the two test blocks $<300 \mathrm{~ms}$ or $>3,000 \mathrm{~ms}$ were replaced with nearer

Table 1 Mean and standard deviation (in brackets) of ownership and agency ratings, and IAT $d$ scores

\begin{tabular}{llllll}
\hline & Ownership & Agency & preIAT & post IAT & IAT changes \\
\hline Synchrony & $3.18(1.32)$ & $5.98(0.98)$ & $0.43(0.32)$ & $0.28(0.34)$ & $-0.15(0.34)$ \\
Asynchrony & $1.97(1.00)$ & $2.24(1.43)$ & $0.46(0.33)$ & $0.46(0.30)$ & $0.001(0.35)$ \\
\hline
\end{tabular}


boundary value; (2) in trials with incorrect responses, the reaction time was replaced by the average RT plus $600 \mathrm{~ms}$; (3) the average RT in the congruent condition was subtracted from the average RT in the incongruent condition; (4) standard deviations were calculated across all trials; and (5) IAT $d$ scores were calculated by dividing the RT difference by the standard deviation.

Next, we computed the preIAT and post IAT $d$ scores (see Table 1 and Fig. 2). The preIAT $d$ scores did not differ between the two groups, $t(95)=0.46, p=.648$, confirming that there was no preexperimental group difference in gender-science stereotype. We analyzed the IAT $d$ scores with a repeated analysis of variance (ANOVA), with synchrony (synchronous or asynchronous) as a between factor, and time (pre or post measure) as a within factor. Significant effects were found for time, $F(1,95)=4.24, p=.042, \eta_{\mathrm{p}}{ }^{2}=0.04$; and for the interaction between synchrony and time, $F(1,95)=4.36, p=.039$, $\eta_{\mathrm{p}}{ }^{2}=0.04$; and marginal for synchrony, $p=.063$. We further analyzed whether the post IAT $d$ scores differed between the two groups: a two-tailed $t$ test, $t(95)=2.75, p=.007, d=0.56$, indicated a group difference in gender-science stereotype after the enfacement illusion inducement.

Then, we subtracted the ${ }_{\text {pre }} \mathrm{IAT} d$ score from the post $_{\mathrm{IAT}} d$ score (post IAT $-{ }_{\text {pre }} \mathrm{IAT}$ ) for the synchrony and the asynchrony group separately, so to obtain IAT $d$ score changes. Note that the IAT $d$ score change indicates the effect of synchrony/ asynchrony on the stereotype: positive values indicate an increase of the stereotype while negative values indicate a decrease of the stereotype. A two-tailed independent-samples $t$ test revealed a significant group difference for IAT $d$ score changes, $t(95)=2.08, p=.041, d=0.42$, indicating different impacts in the two groups: While the change was negative for the synchrony group, indicating a substantial decrease of the gender-science stereotype, there was no significant change for

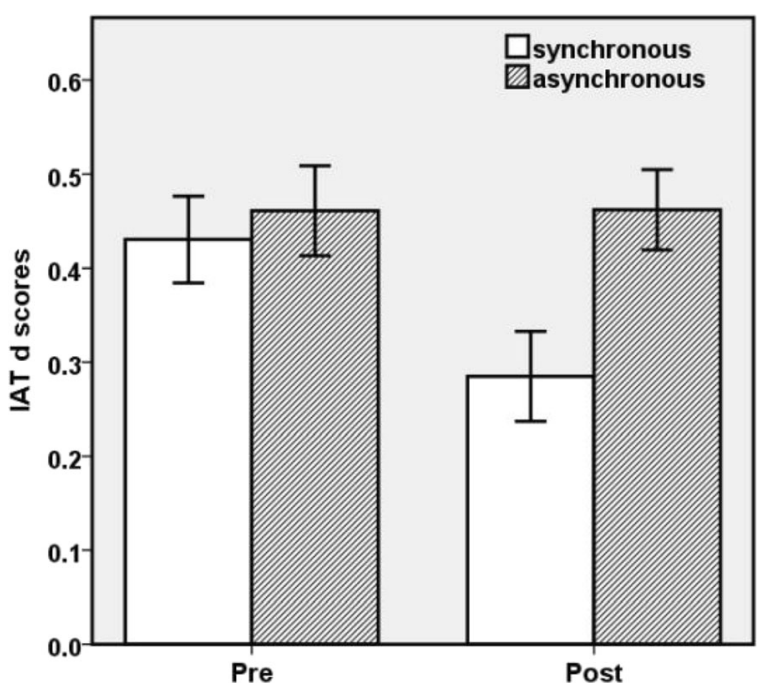

Fig. 2 IAT $d$ scores as a function of time(pre or post measure) and synchrony group. Error bars represent $+/-1 \mathrm{SE}$ the asynchrony group, showing only a small numerical decrease of the stereotype. Testing the $d$ score changes against zero by means of two-tailed $t$ tests showed that the negativegoing change in the synchrony group was significant, $t(48)=$ $3.02, p=.004, d=0.43$, while the positive-going change in the asynchrony group was not, $t(47)=0.02, p=.983$. In other words, synchrony significantly reduced the gender-science stereotype.

IAT $d$ score changes correlated significantly and negatively with aggregated agency scores, $r=-.22, p=.014$, but not with aggregated ownership scores, $r=-.12, p=.119, N=97$, in one-tailed Spearman tests, suggesting that stronger agency over the virtual face came with a weaker gender-science stereotype.

\section{Discussion}

Our aim was to test whether synchronizing the head movements of male participants with the movements of a female avatar head would lead to a relative reduction of the genderscience stereotype. This required the successful induction of ownership over the avatar's face, despite the difference in gender. As shown by the significant impact of synchrony condition on the ownership and agency scores, the induction was successful. It is true that perceived ownership was not perfect, as indicated by the fact that scores were lower than the middle score of 4. This finding is consistent with other studies of the enfacement illusion (Ma et al., 2016; Sforza et al., 2010; Tajadura-Jiménez, Longo, Coleman, \& Tsakiris, 2012) and presumably reflects the fact that, in contrast to the RHI and VHI designs, a short practice over $2 \mathrm{~min}$ was not sufficient to affect one's identification with another face (Tsakiris, 2008). Nevertheless, the significant effect confirmed that synchrony did increase the perceived overlap with, and the greater embodiment of the virtual face with the opposite gender.

For one, this finding is consistent with previous observations showing that participants experienced ownership for other people's faces of the same gender (Ma et al., 2016; Ma et al., 2019; Sforza et al., 2010) and for other people's bodies of a different gender (Tacikowski et al., 2020). As faces are assumed to be the most distinctive feature of people's bodies (Tsakiris, 2008), the fact that participants can integrate a virtual face indicating a different gender into their selfrepresentation confirms the assumption that self-other boundaries can be very fluid indeed.

For another, the finding that the male participants integrated the female faces into their self-representation implies that the preconditions for changing the gender-science stereotype were given. Indeed, synchrony had the opposite effect on stereotyping than asynchrony by showing a significant reduction of the strength of the stereotype as assessed by the IAT. This finding is also consistent with previous studies showing 
that identifying with others can affect one's social perception (Mazzurega et al., 2011; Paladino et al., 2010) and, more importantly, implicit attitudes towards others (Jeong et al., 2019; Maister et al., 2013; Peck et al., 2013). More generally speaking, our findings are also consistent with the idea that synchronizing with the behavior of another person does not only tend to diminish self-other discrimination, but also to invite a kind of "feature migration" (Ma et al., 2019), so that features actually "belonging" to the other (such as race and other physical attributes or affective states: Ma et al., 2016; Ma et al., 2019) can become attached to one's selfrepresentation.

This kind of feature migration is indeed predicted by recent extensions of the theory of event coding (TEC; Hommel, Colzato, \& van den Wildenberg, 2009; Hommel, Müsseler, Aschersleben, \& Prinz, 2001) to address social representation (Hommel, 2018). According to TEC, people represent themselves and others in terms of event files binding codes of various kinds of perceived features, and feature codes can migrate to other files if and to the degree that the files contain overlapping features and/or people adopt a processing mode promoting integration (Hommel, 2018). In our case, synchrony may have tempted the male participants to falsely bind the feature "female" to their current self-representation, which in turn would suggest the interpretation of themselves as being female (Tacikowski et al., 2020). As this feature would now coexist with the knowledge of having a positive relationship with STEM (taken from their male experience), this would lead to a conflict that was apparently reduced by downplaying the gender-science stereotype (Dong, 2019; Wang, 2010). Thus, the present study provides evidence that changes of self-representation can change social judgment. Here we induced these changes by means of synchrony with an avatar, but other methods are likely to exist. For instance, engaging in divergent thinking and being exposed to a relaxing scent (both conditions assumed to promote an integrative processing mode; Hommel \& Colzato, 2017) has been shown to increase interpersonal trust (Sellaro, Hommel, de Kwaadsteniet, \& Colzato, 2014a; Sellaro, van Dijk, Rossi Paccani, Hommel, \& Colzato, 2014b), and engaging in divergent thinking to increase self-other integration (Colzato, van den Wildenberg, \& Hommel, 2013).

Interestingly, the correlations showed a tighter relationship between the IAT $d$ score changes and agency than between the IAT and the ownership ratings, even though agency and ownership were strongly correlated. This is to some extent consistent with findings of our previous study (Ma et al., 2016), in which only agency ratings correlated with changes in selfother integration and mood, and with the observation that synchrony, but not ownership ratings, positively predict the implicit attitude towards others assessed by means of IAT (Jeong et al., 2019). These patterns suggest that agency and ownership ratings rely on overlapping but not completely identical informational resources, and that the relationship between agency and gender stereotype relies on information that is not shared with ownership. Indeed, we and others have suggested that agency and ownership judgments integrate various sources of information (Hommel, 2015; Synofzik, Vosgerau, \& Newen, 2008), including bottom-up information, as provided by sensory synchrony, and top-down information, such as general expectations and background knowledge (Ma \& Hommel, 2015). We assume that the strong connection between agency and ownership emerges from substantial informational overlap regarding bottom-up cues provided by visuomotor synchrony (Botvinick \& Cohen, 1998; Ma \& Hommel, 2015; Synofzik et al., 2008). Interestingly, in work on illusions that are not including the face, such as the RHI or the full body illusion, social judgments have been shown to be sensitive to both agency and ownership, and ownership ratings have been demonstrated to predict IAT score changes (Maister et al., 2013) and the implicit sense of own gender (Tacikowski et al., 2020). However, the human face has been argued to be particularly indicative of one's selfrepresentation (Tsakiris, 2008), so that it makes sense to speculate that involving the face might boost top-down effects on perceived ownership. If so, it might have been these top-down effects on ownership measures that prevented a stronger correlation between ownership and IAT scores. However, more research on this issue is certainly required to draw firmer conclusions.

Apart from its theoretical relevance, our findings also have practical implications. Gender-science stereotypes stand in the way of personal development and societal productivity, which invites efforts to overcome it effectively. On the one hand, the experimental procedure of synchronizing people's behavior with the behavior of an artificial agent is certainly too artificial for practical purposes. On the other hand, however, our theoretical perspective suggests that synchronization is only one of many possible ways to create feature overlap between individuals. If so, any intervention that increases the perceived feature overlap between males and females may also contribute to overcoming stereotypes like the one related to gender and science.

Author contribution X.Z. and K.M. developed the study concept, and all authors contributed to the study design. K.M. programmed the script, X.Z. tested the participants and collected the data. X.Z. and K.M. performed the data analyses, X.Z. and K.M. drafted the manuscript, and B.H. provided critical revisions. All authors approved the final version of the manuscript for submission.

Funding This research was supported by National Natural Science Foundation of China (31700942); the Fundamental Research Funds for the Central Universities (SWU2009429); an experimental technology research project in Southwest University (SYJ2019036) to K.M. in China, and a grant of the German Research Council (DFG: HO 1430/12-1) to B.H. 


\section{Compliance with ethical standards}

All procedures performed in this study were in accordance with the ethical standards of ethics committee at Southwest University and with the 1964 Helsinki declaration and its later amendments. Informed consents were obtained from all participants included in this study.

Conflict of interest The authors declared that they had no conflicts of interest with respect to their authorship or the publication of this article.

None of the data or materials for the experiments reported here is available, and none of the experiments was preregistered.

\section{References}

Botvinick, M., \& Cohen, J. (1998). Rubber hands 'feel' touch that eyes see. Nature, 391(6669), 756. https://doi.org/10.1038/35784

Cheryan, S., Master, A., \& Meltzoff, A. N. (2015). Cultural stereotypes as gatekeepers: Increasing girls' interest in computer science and engineering by diversifying stereotypes. Frontiers in Psychology, 6, 49. https://doi.org/10.3389/fpsyg.2015.00049

Colzato, L.S., van den Wildenberg, W., \& Hommel, B. (2013). Increasing self-other integration through divergent thinking. Psychonomic Bulletin \& Review, 20, 1011-1016. https://doi.org/10.3758/ s13423-013-0413-4

Cvencek, D., Meltzoff, A. N., \& Greenwald, A. G. (2011). Math-gender stereotypes in elementary school children. Child Development, 82(3), 766-79. https://doi.org/10.1111/j.1467-8624.2010.01529.x

Dong, B. (2019). Research on influence of mathematical gender stereotype endorsement and mathematical achievements in middle school students (Master's thesis). East China Normal University, Shanghai, China. (in Chinese).

Faul, F., Erdfelder, E., Lang, A. G., \& Buchner, A. (2007). G*Power 3: A flexible statistical power analysis program for the social, behavioral, and biomedical sciences. Behavior Research Methods, 39(2), 175191. https://doi.org/10.3758/bf03193146

Greenwald, A. G., McGhee, D. E., \& Schwartz, J. L. K. (1998). Measuring individual differences in implicit cognition: The implicit association test. Journal of Personality and Social Psychology, 74(6), 1464-1480. https://doi.org/10.1037/0022-3514.74.6.1464

Greenwald, A. G., Nosek, B. A., \& Banaji, M. R. (2003). Understanding and using the implicit association test: I. An improved scoring algorithm. Journal of Personality and Social Psychology, 85(2), 197216. https://doi.org/10.1037/0022-3514.85.2.197

Hommel, B. (2013). Ideomotor action control: On the perceptual grounding of voluntary actions and agents. In W. Prinz, M. Beisert, \& A. Herwig (Eds.), Action science: Foundations of an emerging discipline (pp. 113-136). Cambridge, MA: MIT Press.

Hommel, B. (2015). Action control and the sense of agency. In P. Haggard \& B. Eitam (Eds.), The sense of agency (pp. 307-326). New York: Oxford University Press.

Hommel, B. (2018). Representing oneself and others: An event-coding approach. Experimental Psychology, 65, 323-331. https://doi.org/ 10.1027/1618-3169/a000433

Hommel, B., \& Colzato, L.S. (2017). The social transmission of metacontrol policies: Mechanisms underlying the interpersonal transfer of persistence and flexibility. Neuroscience and Biobehavioral Reviews, 81, 43-58. https://doi.org/10.1016/j. neubiorev.2017.01.009

Hommel, B., Colzato, L. S., \& van den Wildenberg, W. P. M. (2009). How social are task representations? Psychological Science, 20, 794-798. https://doi.org/10.1111/j.1467-9280. 2009.02367.x
Hommel, B., Müsseler, J., Aschersleben, G., \& Prinz, W. (2001). The theory of event coding (TEC): A framework for perception and action planning. Behavioral \& Brain Sciences, 24, 849-878. https://doi.org/10.1017/s0140525x01000103

Jeannerod, M. (2003). The mechanism of self-recognition in humans. Behavioural Brain Research, 142(1/2), 1-15. https://doi.org/10. 1016/s0166-4328(02)00384-4

Jeong, J. W., Chang, E., \& Kim, H. T. (2019). Multisensory facial stimulation implicitly improves evaluations of the goodness of attractive others. Frontiers in Psychology, 10, 1239. https://doi.org/10.3389/ fpsyg.2019.01239

Ma, K., \& Hommel, B. (2013). The virtual-hand illusion: Effects of impact and threat on perceived ownership and affective resonance. Frontiers in Psychology, 4, 604. https://doi.org/10.3389/fpsyg. 2013.00604

Ma, K., \& Hommel, B. (2015). The role of agency for perceived ownership in the virtual hand illusion. Consciousness and Cognition, 36, 277-288. https://doi.org/10.1016/j.concog.2015.07.008

Ma, K., Sellaro, R., \& Hommel, B. (2019). Personality assimilation across species: Enfacing an ape reduces own intelligence and increases emotion attribution to apes. Psychological Research, 83, 373-383. https://doi.org/10.1007/s00426-018-1048-x

Ma, K., Sellaro, R., Lippelt, D. P., \& Hommel, B. (2016). Mood migration: How enfacing a smile makes you happier. Cognition, 151, 5262. https://doi.org/10.1016/j.cognition.2016.02.018

Maister, L., Sebanz, N., Knoblich, G., \& Tsakiris, M. (2013). Experiencing ownership over a dark-skinned body reduces implicit racial bias. Cognition, 128(2), 170-178. https://doi.org/10.1016/j. cognition.2013.04.002

Maister, L., Slater, M., Sanchez-Vives, M. V., \& Tsakiris, M. (2015). Changing bodies changes minds: Owning another body affects social cognition. Trends in Cognitive Sciences, 19, 6-12. https://doi. org/10.1016/j.tics.2014.11.001

Mazzurega, M., Pavani, F., Paladino, M. P., \& Schubert, T.W. (2011). Self-other bodily merging in the context of synchronous but arbitrary-related multisensory inputs. Experimental Brain Research, 213(2/3), 213-221. https://doi.org/10.1007/s00221-0112744-6

Nosek, B. A., Greenwald, A. G., \& Banaji, M. R. (2005). Understanding and using the implicit association test: II. Method variables and construct validity. Personality and Social Psychology Bulletin, 31(2), 166-180. https://doi.org/10.1177/0146167204271418.

Nosek, B. A., Smyth, F. L., Sriram, N., Lindner, N. M., Devos, T., Ayala, A., Bar-Anan, Y., Bergh, R. Cai, H., Gonsalkorale, K., Kesebir, S., Maliszewski, N., Neto, F., Olli, E., Park, J., Schnabel, K., Shiomura, K., Tulbure, B. T., Wiers, R. W., Somogyi, M., Akrami, N., Ekehammar, B., Vianello, M., Banaji, M. R., \& Greenwald A. G. (2009). National differences in gender-science stereotypes predict national sex differences in science and math achievement. Proceedings of the National Academy of Sciences of the United States of America, 106(26), 10593-10597. https://doi.org/10.1073/ pnas.0809921106

Paladino, M. P., Mazzurega, M., Pavani, F., \& Schubert, T. W. (2010). Synchronous multisensory stimulation blurs self-other boundaries. Psychological Science, 21, 1202-1207. https://doi.org/10.1177/ 0956797610379234

Peck, T. C., Seinfeld, S., Aglioti, S. M., \& Slater, M. (2013). Putting yourself in the skin of a black avatar reduces implicit racial bias. Consciousness and Cognition, 22(3), 779-787. https://doi.org/10. 1016/j.concog.2013.04.016

Ramachandran, V. S. (1998). Consciousness and body image: lessons from phantom limbs, Capgras syndrome and pain asymbolia. Philosophical Transactions of the Royal Society B: Biological Sciences, 353, 1851-1859. https://doi.org/10.1098/rstb.1998.0337

Sanchis-Segura, C., Aguirre, N., Cruz-Gómez, A. J., Solozano, N., \& Forn, C. (2018). Do gender-related stereotypes affect spatial 
performance? Exploring when, how and to whom using a chronometric two-choice mental rotation task. Frontiers in Psychology, 9, 1261. https://doi.org/10.3389/fpsyg.2018.01261

Sellaro, R., Derks, B., Nitsche, M. A., Hommel, B., Den Wildenberg, W. P., Van Dam, K., \& Colzato, L. S. (2015). Reducing prejudice through brain stimulation. Brain Stimulation, 8(5), 891-897. https://doi.org/10.1016/j.brs.2015.04.003

Sellaro, R., Hommel, B., de Kwaadsteniet, E. W., \& Colzato, L. S. (2014a). Increasing interpersonal trust through divergent thinking. Frontiers in Psychology, 5, 561. https://doi.org/10.3389/fpsyg. 2014.00561

Sellaro, R., van Dijk, W. W., Rossi Paccani, C., Hommel, B., \& Colzato, L. S. (2014b). A question of scent: lavender aroma promotes interpersonal trust. Frontiers in Psychology, 5, 1486. https://doi.org/10. 3389/fpsyg.2014.01486

Sforza, A., Bufalari, I., Haggard, P., \& Aglioti, S. M. (2010). My face in yours: Visuo tactile facial stimulation influences sense of identity. Social Neuroscience, 5, 148-162. https://doi.org/10.1080/ 17470910903205503

Smyth, F. L., \& Nosek, B. A. (2015). On the gender-science stereotypes held by scientists: explicit accord with gender-ratios, implicit accord with scientific identity. Frontiers in Psychology, 6, 415. https://doi. org/10.3389/fpsyg.2015.00415

Steegh, A., Höffler, T., Höft, L., \& Parchmann, I. (2020). First steps toward gender equity in the chemistry Olympiad: Understanding the role of implicit gender-science stereotypes. Journal of Research in Science Teaching, 58(1), 40-68. https://doi.org/10. $1002 /$ tea.21645

Synofzik, M., Vosgerau, G., \& Newen, A. (2008). I move, therefore I am: A new theoretical framework to investigate agency and ownership. Consciousness and Cognition, 17, 411-424. https://doi.org/10. 1016/j.concog.2008.03.008
Tacikowski, P., Fust, J., \& Ehrsson, H. H. (2020). Fluidity of gender identity induced by illusory body-sex change. Scientific Reports 10(1), 14385. https://doi.org/10.1038/ s41598-020-71467-z

Tajadura-Jiménez, A., Longo, M. R., Coleman, R., \& Tsakiris, M. (2012). The person in the mirror: using the enfacement illusion to investigate the experiential structure of self-identification. Consciousness and Cognition, 21(4), 1725-1738. https://doi.org/ 10.1016/j.concog.2012.10.004

Treisman, A. M., \& Gelade, G. (1980). A feature-integration theory of attention. Cognitive Psychology, 12(1), 97-136. https://doi.org/10. 1016/0010-0285(80)90005-5

Tsakiris, M. (2008). Looking for myself: Current multisensory input alters self-face recognition. PLOS ONE, 3(12), e4040.

Vick, S. B., Seery, M. D., Blascovich, J., \& Weisbuch, M. (2008). The effect of gender stereotype activation on challenge and threat motivational states. Journal of Experimental Social Psychology, 44(3), 624-630. https://doi. org/10.1016/j.jesp.2007.02.007

Wang, P., (2010). The development of middle school students' mathematical gender stereotype, identity of sex role and their impact on mathematical achievement (Master's thesis). East China Normal University, Shanghai, China. (in Chinese). https://doi.org/10.7666/ d.y 1741874

$\mathrm{Xu}, \mathrm{Z}$. , (2012). The gender science stereotype among high school students and intervention (Master's thesis). Nanjing Normal University, Nanjing, China. (in Chinese).

Publisher's note Springer Nature remains neutral with regard to jurisdictional claims in published maps and institutional affiliations. 